\title{
Wide complex tachycardia in ischemic heart disease patient: What is the rhythm?
}

\section{Ghaliah Al Mohani*}

Sr Consultant National Heart center, Muscat, Oman

An 84 year-old man presented to Emergency department complaining of cough and fever. He was known to be ischemic heart disease with coronary angiogram done in 2011 showed total occlusion of LAD and 50\% Circumflex artery. Recent Echocardiography showed LV EF 35\%. No chest pain. He is known to suffer from Parkinsonism non complaint to medications due to hallucination as side effect.

On evaluation the patient was hemodynamically stable and had no complaints. Her pulse was $72 \mathrm{bpm}$ and regular with blood pressure 128/76, ECG showed q wave in anterior leads with no ST changes (Figure 1). He was kept under observation and monitor. After coming from wash room, patient developed ventricular arrhythmias, hemodynamically stable. 12 lead ECG (figure 2) was performed and amiodarone was given with successful resumption of sinus rhythm as reported by medical staff in emergency department. Such diagnosis was confirmed by cardiologist and patient was shifted to cardiac intermediate care unit for further monitor and management.

The possibility of artifact as a cause of ECG findings should always be considered in an otherwise asymptomatic patient who is hemodynamically stable. In our patient, a cursory overview of the ECG suggested ventricular tachycardia. However, close scrutiny, particularly of lead V1 and V5 on Figure 2, clearly shows the QRS complexes buried in the wide amplitude, repetitive electrical activity. Tremor-induced ECG artifact should always be considered as a cause of bizarre ECG changes. The ECG should always be interpreted in the context of the patient's condition and any other extraneous factors present at the time of the recording.

Characteristics that differentiate artifact from ventricular tachycardia include the absence of hemodynamic deterioration during

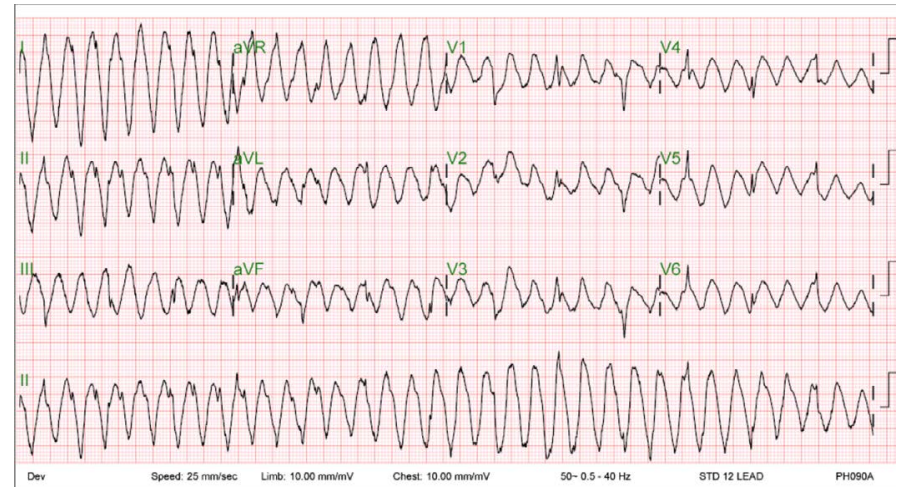

Figure 1. ECG of the patient once back from wash room.

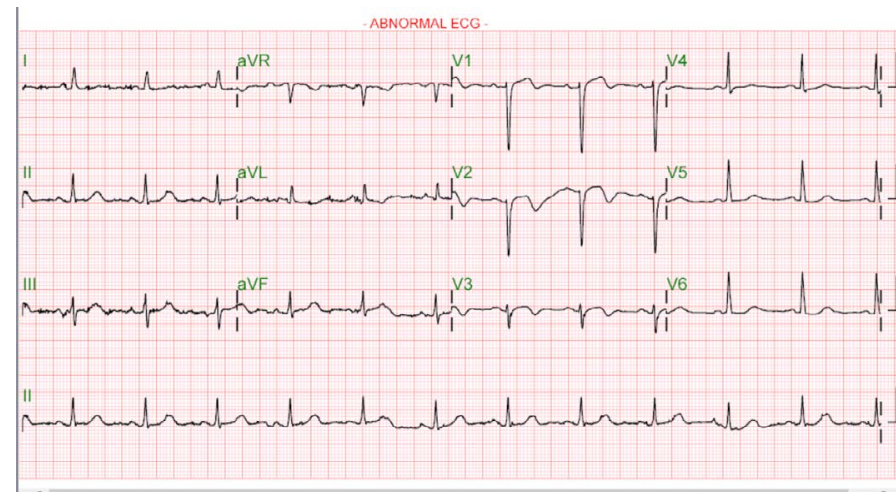

Figure 2. ECG of the patient after few minutes to document by the physician resumption of Sinus Rhythm

the event; normal QRS complexes within the artifact; an unstable base line on the electrocardiogram before the event, after the event, or both; and an association with body movement. In this case, the artifact that mimicked ventricular tachycardia and resulted in unnecessary intervention was usually not associated with symptoms and could be distinguished from ventricular tachycardia by the presence of QRS complexes that was visible in the electrocardiographic artifact at intervals that coincided with the cycle length of the base-line rhythm. The presence of history of Parkinsonism should be considered as important cause of tremor induced artifact ECG [1].

Although the magnitude of the problem remains unclear, this case in this report demonstrate that physicians with a broad spectrum of expertise in the diagnosis and treatment of arrhythmia, including board-certified cardiologists, at times may mistake artifact for ventricular tachycardia. Furthermore, this case show that the misdiagnosis of electrocardiographic artifact as ventricular tachycardia may lead to unnecessary interventions as drastic as the implantation of an implantable cardioverter-defibrillator. These findings indicate the importance of improved training in the recognition of artifact and the need for a heightened index of suspicion among physicians who treat patients with arrhythmias [2].

Correspondence to: Ghaliah Al Mohani, MD, PHD, CEPS, FESC, FHRS, Sr Consultant National Heart center, Muscat, Oman, E-mail: gmohanny@hotmail. com

Received: January 02, 2018; Accepted: February 15, 2018; Published: February 19,2018 


\section{References}

1. Srikureja W, Darbar D, Reeder GS (2000) Tremor-induced ECG artifact mimicking ventricular tachycardia. Circulation 102: 1337-8. [Crossref]
2. Knight BP, Pelosi F, Michaud GF, Strickberger SA, Morady F (1999) Clinical Consequences of Electrocardiographic Artifact Mimicking Ventricular Tachycardia. $N$ Engl J Med 341: 1270-1274. [Crossref]

Copyright: (C2018 Ghaliah Al Mohani. This is an open-access article distributed under the terms of the Creative Commons Attribution License, which permits unrestricted use, distribution, and reproduction in any medium, provided the original author and source are credited. 